\title{
Normas de categorización, organización y funcionamiento de las Unidades de Cuidados Intensivos e Intermedios Pediátricos en los establecimientos asistenciales Parte I: Categorización - Unidad de Cuidados Intensivos Pediátricos Nivel 1
} Guidelines of categorization, organization and functioning of the Pediatric Intensive Care Units and Pediatric Intermediate Care Units in health care facilities. Part I: Categorization Pediatric Intensive Care Unit Level 1

Comité Nacional de Emergencias y Cuidados Críticos de la Sociedad Argentina de Pediatría y Capítulo Terapia Intensiva Pediátrica-Sociedad Argentina de Terapia Intensiva $a^{b}$

http:/ /dx.doi.org/10.5546/aap.2014.284

\section{INTRODUCCIÓN}

El primer objetivo de la presente norma es mejorar la organización y el funcionamiento de los Servicios o Unidades de Terapia Intensiva Pediátrica (UTIP), ${ }^{1}$ más comunmente denominados de Cuidados Intensivos Pediátricos (UCIP), a fin de lograr estándares mínimos de calidad que permitan un cuidado efectivo de los pacientes pediátricos críticamente enfermos y una disminución de la morbilidad y la mortalidad. El segundo objetivo es presentar una propuesta para la categorización de las UCIP.

\section{CATEGORIZACIÓN DE LAS UNIDADES DE CUIDADOS INTENSIVOS PEDIÁTRICOS}

Las Unidades de Cuidados Intensivos Pediátricos (UCIP) se categorizan de acuerdo con la capacidad para resolver patologías específicas y con los recursos tecnológicos en UCIP Nivel 1 y UCIP Nivel 2., ${ }^{1,2}$

Los establecimientos que habitualmente reciben patologías de bajo riesgo y no tienen UCIP deben contar con una Unidad de Reanimación (UR).

La UCIP 1 debe proveer cuidados multidisciplinarios a los pacientes con enfermedades graves o complejas de diverso origen, que incluyen afecciones médicas, quirúrgicas o traumáticas. La mayoría de las UCIP 1 se localizan en hospitales pediátricos $\mathrm{u}$ hospitales generales de alta complejidad. Deben contar con acceso a especialidades y subespecialidades pediátricas, tanto médicas como quirúrgicas, que
Correspondencia: Dr. Thomas Iolster: tiolster@cas.austral. edu.ar

Conflicto de intereses: Ninguno que declarar.

Recibido: 7-1-2014 Aceptado: 13-1-2014
Coordinadores: Dr. Gustavo Debaisi ${ }^{a}$, Dr. Daniel Capra ${ }^{b}$, Dr. Thomas Iolster ${ }^{a}$, Dr. Luis Landrya, Dr. Pablo Minces ${ }^{a}$, Dra. Gabriela Sheehan ${ }^{b}$ y Dra. María Elena Ratto ${ }^{b}$ Participaron en la discusión y redacción de estas normas los siguientes profesionales: Comité Nacional de Emergencias y Cuidados Críticos - Sociedad Argentina de Pediatría: Dres. Rodolfo Pablo Moreno, Silvia Santos, Mariam Sarli, Daniel Rufach, Silvio Fabio Torres, Daniel Buamscha, Juan Carlos Vassallo. Capítulo Terapia Intensiva Pediátrica - Sociedad Argentina de Terapia Intensiva: Dres. María del Pilar Arias, Analia Fernández, In Ja Ko, Ana María Nieva, Gladys Palacio, Liliana Saligari, Alejandro Siaba Serrate. 
contribuyan a resolver las necesidades fisiológicas y emocionales de niños críticamente enfermos. ${ }^{1,2}$ Dentro de las UCIP 1, se incluyen aquellas unidades que resuelven patologías específicas, como cirugía cardiovascular, trasplante de órganos, pacientes quemados, etc.

Las UCIP 2 proveen cuidados a pacientes con enfermedades críticas, pero pueden no tener los recursos para atender poblaciones de pacientes con patologías específicas, por no contar con los especialistas, áreas de apoyo o equipamiento necesarios para su atención. Estos centros deben tener establecido un proceso para la derivación de estos pacientes con patologías específicas. ${ }^{1,2}$

Las Unidades de Reanimación (UR) deben proveer cuidados a pacientes pediátricos en riesgo para su estabilización hasta que puedan ser derivados en el menor tiempo posible a otra institución de mayor complejidad que cuente con UCIP Nivel 1 o 2.

De acuerdo con estas definiciones, la categorización de las UCIP o de las UR debe estar relacionada con la categorización del establecimiento asistencial. ${ }^{1}$

1. Establecimientos de baja complejidad: la atención pediátrica de pacientes críticos se hará en los servicios de emergencias o en la UR. Deberá contar con un sistema adecuado de traslado de pacientes críticos pediátricos a otra institución de mayor nivel (integrando así una red).

2. Establecimientos de moderada complejidad: constituyen una franja heterogénea en la que el tipo de patología que reciben y que deben asistir depende no solo de la complejidad tecnológica, sino además de la situación geográfica en la que se encuentren y de las necesidades epidemiológicas por cubrir. A criterio de la autoridad sanitaria correspondiente, podrán diseñar UR o bien contar con una UCIP.

3. Establecimientos asistenciales de alta complejidad: deben contar con una UCIP. Los establecimientos de alta complejidad especializados (hospitales pediátricos y materno-infantiles) deberán contar, en todos los casos, con una UCIP de Nivel 1.

Los pacientes con enfermedades crónicas, estables clínicamente, pero dependientes de tecnología (ejemplo: asistencia ventilatoria mecánica prolongada, nutrición parenteral total, etc.) deberían continuar su internación, de manera ideal, en un área específicamente diseñada para su correcto cuidado o bajo un programa de internación domiciliaria.
En el Anexo (versión electrónica) se enumeran los requisitos para una categorización adecuada.

\section{UNIDAD DE TERAPIA INTENSIVA PEDIÁTRICA NIVEL 1}

Es la unidad de internación de pacientes pediátricos (de un mes hasta 15 años de edad). A partir de 16 años y hasta 18 años, los criterios de internación podrán consensuarse entre los servicios de la Unidad de Cuidados Intensivos de Adultos (UCI) y la UCIP de acuerdo con las características del paciente, los antecedentes clínicos y los criterios institucionales. Estos pacientes de mayor edad podrían tener patologías complejas seguidas desde la pediatría que se encuentran en estado crítico actual o potencial, con posibilidades de recuperación parcial o total, que requieran para su supervivencia de servicios integrales de atención médica y de enfermería en forma permanente y constante, además de equipos e instrumental que aseguren el adecuado control del tratamiento del paciente.

Las UCIP Nivel 1 solo podrán funcionar en establecimientos de internación que cumplan los siguientes requisitos:

\section{Especialidades y servicios de apoyo e interconsulta: $:^{2,3}$}

a. Anestesiología con experiencia pediátrica de guardia activa las $24 \mathrm{~h}$.

b. Cirugía Pediátrica con guardia activa o pasiva disponible en la Unidad dentro de los 60 minutos durante las $24 \mathrm{~h}$, siempre que el centro cuente con cirujano general de guardia activa.

c. Neurocirugía con experiencia en la atención de pacientes pediátricos, de guardia activa o pasiva disponible en la Unidad en 60 minutos durante las $24 \mathrm{~h}$.

d. Hemoterapia y banco de sangre, con guardia activa de $24 \mathrm{~h}$ a cargo de personal técnico o Servicio de Medicina Transfusional con acceso rápido a hemocentros regionales. Deben cumplir las normas establecidas por el Programa Nacional de Garantía de Calidad de la Atención Médica sobre Medicina Transfusional (702/93) y estar a cargo de un médico hemoterapeuta. ${ }^{1}$

e. Radiología con servicio de rayos central y equipo portátil en la UTIP, con guardia activa de $24 \mathrm{~h}$ a cargo de personal técnico.

f. Ecografía disponible dentro de los 60 minutos.

g. Tomografía axial computada (TAC) 
disponible en la institución y guardia técnica activa de $24 \mathrm{~h}$. Resonancia magnética nuclear (RMN) disponible en la institución o la posibilidad de realizarla.

h. Laboratorio ubicado dentro de la planta física de la institución, con guardia activa de 24 h. Estará a cargo de un bioquímico. Como mínimo, deberá tener capacidad para realizar análisis programados, estudios infectológicos y de urgencia. Estos últimos incluyen gases en sangre, medio interno, hematología, coagulación y funciones parenquimatosas básicas, cuyos resultados deben estar disponibles dentro de los 30 minutos. Es recomendable la disponibilidad de autoanalizador de gases, ácido láctico, glucemia, ionograma y hematocrito en sangre dentro de la Unidad.

i. Endoscopia digestiva y respiratoria con guardia pasiva de $24 \mathrm{~h}$.

j. Traumatólogo con guardia activa; contar con interconsultor pediátrico.

k. Cardiología pediátrica con guardia pasiva de $24 \mathrm{~h}$.

1. Radiología intervencionista con guardia pasiva de $24 \mathrm{~h}$.

m. Posibilidad de consulta con especialistas pediátricos no considerados en los ítems anteriores.

2. Planta física: $:^{2,3}$

Estará ubicada en una zona de circulación semirrestringida y deberá contar con:

a. Superficie: no menor de $7 \mathrm{~m}^{2}$ por cama en áreas abiertas y no menos de $9 \mathrm{~m}^{2}$ en habitaciones individuales, con un área de superficie total de la unidad equivalente al doble de la superficie destinada a las camas. El 50\% del área total de la UCIP corresponde a las áreas de apoyo.

b. Número de camas: no debe ser menor de seis y es recomendable que estas Unidades mantengan una actividad mínima de, por lo menos, 1000 días/pacientes anuales. En situaciones especiales, podrá considerarse un número menor de camas (cuatro) para unidades especializadas en cirugía cardiovascular, trasplante o quemados.

c. Debe contar con espacio para aislamiento de pacientes.

3. Áreas de apoyo: $:^{2,3}$

Debe contar con:

1. Estación de enfermería.
2. Sala de estar para enfermería y personal de limpieza.

3. Depósito de equipos.

4. Depósito de ropa y material de uso diario limpio (ropa, material quirúrgico, etc.).

5. Stock de medicamentos y material descartable. Refrigerador para medicación, compartimentos bajo llave para narcóticos y electrolitos de alta concentración. Refrigerador independiente para fórmulas de alimentación.

6. Depósito de ropa y material sucio y de desecho. Este debe estar en el sector de salida, dentro del esquema de tráfico de la UCIP, o tener circulación independiente. El traslado de desechos debe cumplir las especificaciones de la Ley $\mathrm{N}^{\mathrm{o}} 24051 /$ Decreto reglamentario 831/93, Resolución del Boletín Oficial 27669. Idealmente, debería contar con un refrigerador para material biológico.

7. Área de lavado y preparación para material.

8. Oficinas administrativas y/o de secretaría.

9. Área de recepción de los familiares de los pacientes internados. De acuerdo con las características de cada establecimiento, podrá implementarse una sala de estar para los familiares de los niños internados. Tendrá que contar con instalaciones sanitarias adecuadas. Es aceptable que este espacio se encuentre fuera del ámbito hospitalario, cercano a él.

10. Área de reunión de médicos de planta y de enfermería dentro de la UTIP o adyacente a ella. Es recomendable contar con un lugar privado para facilitar el diálogo con los familiares de los pacientes.

11. Oficina del jefe/a médico y del jefe/a de Enfermería de la Unidad.

12. Habitación con cama para médicos de guardia próxima a la UCIP con baño completo (lavabo, inodoro y ducha).

13. Baños próximos para el personal y chatero.

14. Se recomienda contar con accesorios que contribuyan a amenizar la internación (ejemplo: televisión, etc.).

15. Medidas de protección contra incendios y plan de evacuación de acuerdo con las normativas vigentes, con su correspondiente señalización.

16. Es deseable contar con un espacio que permita la intimidad de la familia con el niño recientemente fallecido. 
17. Acceso a internet y biblioteca con fines docentes y asistenciales.

\section{4. Áreas de internación:2,3}

Debe contar con:

a. Pileta para lavado de manos en el ingreso a la unidad: no menos de una cada cuatro camas, con canillas operadas preferentemente con el pie o el codo o célula fotoeléctrica y de suficiente profundidad para evitar salpicar en los alrededores. Debe implementarse, además, un sistema de alcohol-gel para la higiene de manos en cada cama.

b. De ser posible, se recomienda contar con ventanas al exterior para dar luz natural y orientación témporo-espacial al paciente. Solo podrían abrirse en caso de emergencia.

c. Paredes lavables pintadas de color que no provoque sensación depresiva ni que sean excitantes por el brillo. Piso con cobertura antideslizante, lavable y con zócalo sanitario.

d. Visualización directa por parte del personal de enfermería de los pacientes desde la central o por otros medios ópticos, desde el pasillo de la UCIP o desde la cama de otro paciente. Otra opción es el monitoreo central de funciones vitales con vigilancia a través de cámaras de video o una pantalla con los parámetros de cada paciente.

e. Enchufes en cantidad necesaria y no menos de diez por cama con voltaje y amperaje de acuerdo con las especificaciones técnicas locales que se deben ubicar a $70 \mathrm{~cm}$ del piso para facilitar la conexión y evitar la desconexión con el pie. Enchufe y línea eléctrica para equipo de rayos $x$ portátil. Sistema de alimentación eléctrica de emergencia (UPS), que asegure el suministro ininterrumpido. Los sistemas de enchufe deben cumplir con las normas de seguridad eléctrica vigentes.

f. Dos salidas de oxígeno, una de aire comprimido y, al menos, una de aspiración, preferentemente dos.

g. Iluminación adecuada, consistente en iluminación general de techo y luz nocturna que permita descansar al paciente. Debe contarse con lámparas de gran intensidad (aproximadamente, lúmenes equivalentes a $200 \mathrm{~W}$ ) para la realización de procedimientos específicos sobre el paciente, de brazo movible o similar. Luz de emergencia. h. Aire convenientemente climatizado y filtrado según las normas vigentes.

i. Puertas con abertura que permita el paso de camillas y equipos.

j. Alarma de paro.

5. Equipamiento instrumental e insumos de uso exclusivo para la UCIP,

a. Stock de medicamentos, material descartable e insumos, que cubra las necesidades de $24 \mathrm{~h}$, de las patologías propias de ser tratadas en Terapia Intensiva, para el total de las camas de la Unidad.

b. Un monitor multiparamétrico (ECG, presiones invasiva y no invasiva, temperatura y oximetría de pulso) por cama.

c. Capnógrafo disponible cada cuatro pacientes respirados.

d. Respiradores para técnicas de ventilación convencional: su número no debe ser menor del $70 \%$ de las camas, aunque es deseable disponer de uno por cama; el $80 \%$ de los equipos deben ser microprocesados y con capacidad para medir la mecánica pulmonar. Es aconsejable contar con, al menos, un respirador de alta frecuencia en la Unidad.

e. Carro de reanimación: uno en cada sector. Este incluirá elementos para intubación endotraqueal (tubos endotraqueales de varios diámetros, laringoscopio pediátrico y de adultos con ramas rectas y curvas, mandril, tubos de Mayo, pinza de Magyll, máscaras laríngeas para niños y adultos, sondas de aspiración oro-nasogástricas y nasotraqueales, bolsa de reanimación y máscaras (neonatal, pediátrica y adulta), soluciones y medicamentos para la reanimación avanzada (normas PALS). Equipo de desfibrilación y cardioversión (este podrá estar emplazado en una mesa de transporte aparte). Aguja para acceso intraóseo. Equipo para cricotirotomía.

f. Bombas de infusión de precisión (desde 0,1 hasta $999 \mathrm{ml} / \mathrm{h}$ ): un mínimo de cuatro por cada cama.

g. Bombas de alimentación enteral: una cada dos camas. Estas pueden reemplazarse con las bombas mencionadas en el punto anterior.

h. Electrocardiógrafo: uno por Unidad.

i. Marcapaso externo transitorio cada tres camas en las Unidades de recuperación cardiovascular y uno disponible en la unidad polivalente. 
j. Bolsa de resucitación con reservorio y máscara en cada cama.

k. Equipo para cateterización venosa percutánea de 4 a 7 Fr., dos disponibles de cada tamaño.

1. Equipo para acceso intraóseo, al menos uno por unidad (ubicado en el carro de reanimación).

$\mathrm{m}$. Se debe contar con los recursos para medir volumen minuto cardíaco o alguno de sus subrogantes, por termodilución o por técnica mínimamente invasiva.

n. Equipo específico para monitoreo de presión intracraneana (PIC).

o. Equipos para disección venosa, drenaje pleural, punción lumbar y diálisis peritoneal.

p. Acceso a hemodiálisis. Es conveniente contar con instalaciones de tuberías hídricas correspondientes, al menos, en una cama.

q. Hemodiafiltración continua o equivalente.

r. Ecógrafo portátil disponible.

s. Ecocardiógrafo Doppler color disponible.

t. Disponibilidad de radiografía portátil en la unidad las $24 \mathrm{~h}$.

u. Disponibilidad de EEG las $24 \mathrm{~h}$.

v. Disponibilidad de cama/camilla de transporte con monitor multiparamétrico portátil. Es recomendable contar con un respirador portátil y capnografía de transporte.

w. Oxígeno para transporte. Recomendable, de aluminio.

x. Para aquellas Unidades que atiendan pacientes con patología neurológica crítica, se aconseja disponer de ecografía Doppler transcraneal.

y. Equipo para regulación térmica del paciente.

z. Sistemas para prevención de escaras.

\section{Recursos humanos: $:^{1,2,-6}$}

\section{a. Médicos}

1.Jefe de Servicio de Cuidados Críticos. Médico pediatra especialista en Terapia Intensiva Pediátrica, certificado por autoridad competente o entidad de ley. La dedicación deberá ser como mínimo de seis horas diarias y no menos de $30 \mathrm{~h}$ semanales.

2. Jefe de Unidad o subjefe de servicio o sala o coordinador de Cuidados Intensivos: médico pediatra especialista en Terapia
Intensiva Pediátrica, certificado por autoridad competente o entidad de ley. La dedicación deberá ser como mínimo de seis horas diarias y no menos de $30 \mathrm{~h}$ semanales.

3. Médicos de planta (en unidades de seis o menos camas, este puede ejercer la función del ítem anterior): un médico cada cuatro camas habilitadas o fracción menor. La dedicación deberá ser, como mínimo, de seis horas diarias y no menos de 30 h semanales. Serán médicos pediatras especialistas en Terapia Intensiva Pediátrica, certificados por autoridad competente o entidad de ley.

4.Médico de Guardia: activa en el servicio durante las $24 \mathrm{~h}$, permanente y exclusivo para la Unidad. La dotación mínima será de un médico cada seis camas o fracción. Superando este número, deberá agregarse otro profesional cada seis camas o fracción menor. Deberá contar con la especialidad de Terapia Intensiva Pediátrica, certificada por autoridad competente o entidad de ley o, al menos, dos años de experiencia certificada (beca o residencia) en una Unidad de Terapia Intensiva Pediátrica. Aun cuando los médicos de guardia deberían cumplir todos los requisitos arriba expuestos, ante la carencia de recursos humanos en la especialidad, se acepta que el segundo médico de guardia sea un becario o residente de la especialidad, con probada capacidad para manejar la emergencia pediátrica, con el aval del jefe del Servicio.

En aquellos servicios que tengan establecido un sistema de guardias pasivas con disponibilidad de un especialista dentro de los 30 minutos, podrá considerarse que el tercer médico sea un residente avanzado de otra especialidad. La modalidad de guardia pasiva no debe considerarse obligatoria. Será exigible que cuente con el curso de resucitación avanzada pediátrica PALS, aunque es recomendable haber aprobado otros cursos, como el PFCCS, ATLS, AITP, cursos de ventilación mecánica pediátrica y otros que a futuro puedan ser avalados y/o desarrollados por las sociedades científicas. Es deseable que los médicos que realizan guardias activas concurran a la Unidad fuera de los horarios de la guardia. 
Sería deseable en el futuro contar con tres niveles médicos: jefe, coordinador y médico con función asistencial; esta última cuenta con horario de planta (de 8 a 12 h diarias), para asegurar continuidad en la atención de los pacientes, y horario de guardia.

La relación médico/cama será de 1/6 o fracción menor. Los médicos que cubren el horario de guardia deben contar con los requerimientos formativos del punto anterior. $^{3}$

\section{b. Enfermería}

1.Jefe de Enfermería: enfermero/a profesional o licenciado/a, con un mínimo de dos años de experiencia en un Servicio de Terapia Intensiva Pediátrica y con una dedicación no menor de 35 h semanales.

2. Referente de turno de enfermería: ídem b1, uno por turno de enfermería.

3. Enfermero/a de planta: activo, permanente y exclusivo para la Unidad. Un enfermero cada dos camas o fracción, por turno, con conocimiento y experiencia en Terapia Intensiva Pediátrica.

Se recomienda que el personal esté certificado para la reanimación de pacientes pediátricos por entidades reconocidas. Es recomendable que tenga la licenciatura en la profesión.

Sobre la base de la carga de atención de enfermería, esta relación se puede incrementar a 1 enfermera cada paciente y medio o aun a 1 a 1 . Es recomendable adecuar la carga de atención de enfermería a un puntaje (TISS 28 o similar).

c. Kinesiólogo

Uno disponible por unidad, con guardia activa las $24 \mathrm{~h}$, con experiencia comprobable para el cuidado respiratorio y rehabilitación motora de los pacientes críticos; se recomienda la formación a través de becas, residencias y cursos de posgrado en entidades reconocidas. Se recomienda contar con un kinesiólogo cada 8 camas o fracción, activo y exclusivo (planta o guardia) para la Unidad de 8 a $20 \mathrm{~h}$, y queda a criterio de la Unidad la proporción entre las 20 y las $8 \mathrm{~h}$, que no debe ser menor del $50 \%$ de la dotación diurna.

d. Salud mental: psicólogo o psiquiatra disponible para la Unidad.

e. Servicio social: trabajador social disponible para la Unidad.

f. Personal de limpieza: disponible las 24 h, que garantice el cumplimiento de las normas vigentes de higiene hospitalaria.

g. Personal de apoyo: se recomienda contar con una persona para las tareas auxiliares del servicio disponible las $24 \mathrm{~h}$.

Se recomienda contar con un acompañamiento espiritual si la familia lo requiriese.

h. Personal administrativo: secretaria de servicio.

i. Farmacéutico: disponible las $24 \mathrm{~h}$ en la institución. Se recomienda contar con un farmacéutico clínico disponible para la Unidad, para el adecuado control y supervisión de los medicamentos.

7. Registros ${ }^{1,2}$

Serán exigibles los siguientes registros:

a. Diagnóstico y fecha del ingreso/egreso; datos demográficos (fecha de nacimiento, edad en meses, procedencia, sexo).

b. Tasa de mortalidad estandarizada: mortalidad observada/mortalidad esperada (a la fecha de la confección de este documento, debe utilizarse el PIM 2).

c. Condiciones de egreso: vivo, muerto.

d. Registros de infecciones adquiridas en la Unidad (neumonía asociada a respirador, infección asociada a catéteres venosos centrales e infección urinaria asociada a sonda vesical, por 1000 días de uso del dispositivo). Estos datos deberán ser comunicados a la entidad de control que corresponda.

e. Eventos adversos: extubación no programada por cada 100 días de uso, úlceras por presión, caídas de la cama.

f. Cada Unidad deberá registrar, al menos, un indicador de procesos.

8. Normas de procedimientos y tratamiento en la UCIP

a. Criterios de admisión y egresos.

b. Guías de diagnóstico y tratamiento para la patología habitual de la UCIP.

c. Protocolos de atención de enfermería.

d. Es recomendable poseer un programa de evaluación, monitorización y mejora continua de la calidad, así como la implementación de un programa para la atención segura de los pacientes.

e. Política de consentimiento informado para los procedimientos.

f. Control de infecciones. La Unidad deberá contar con un programa de control 
de infecciones que incluya normas de limpieza, de lavado de manos, de manejo de material sucio y contaminado de acuerdo con la legislación vigente.

g. Protocolos para los procedimientos invasivos.
h. Normas de atención del paro cardiorrespi- ratorio.

\section{BIBLIOGRAFÍA}

1. Ministerio de Salud de la Nación, Programa Nacional de Garantía de la Calidad de la Atención Médica. Normas de Organización y Funcionamiento de Unidades de Cuidados Intensivos Pediátricos. Boletín Oficial N. ${ }^{\circ} 29.779$, $1^{\text {a }}$ sección, Resolución 1331/01, del 21 de noviembre del 2001. [Consulta: 2 de octubre de 2012]. Disponible en: http:/ / www.msal.gov.ar/pngcam/resoluciones / msres1331_2001.pdf.

2. Rosenberg DI, Moss MM, American College of Critical Care Medicine of the Society of Critical Care Medicine. Guidelines and levels of care for pediatric intensive care units. Crit Care Med 2004;32(10):2117-27.

3. Andrews P, Azoulay E, Antonelli M, Brochard L, et al. Year in review in intensive care medicine, 2004. III. Outcome, ICU organisation, scoring, quality of life, ethics, psychological problems and communication in the ICU, immunity and hemodynamics during sepsis, pediatric and neonatal critical care, experimental studies. Intensive Care Med 2005;31(3):356-72.
4. Pollack MM, Patel KM, Ruttimann E. Pediatric critical care training programs have a positive effect on pediatric intensive care mortality. Crit Care Med 1997;25(10):1637-42.

5. Treggiari MM, Martin DP, Yanez ND, Caldwell E, et al. Effect of intensive care unit organizational model and structure on outcomes in patients with acute lung injury. Am J Respir Crit Care Med 2007;176(7):685-90.

6. Hixson ED, Davis S, Morris S, Harrison AM. Do weekends or evenings matter in a pediatric intensive care unit? Pediatr Crit Care Med 2005;6(5):523-30.

7. American Academy of Pediatrics Committee on Pediatric Emergency Medicine; American College of Emergency Physicians Pediatric Committee; Emergency Nurses Association Pediatric Committee. Joint policy statement-guidelines for care of children in the emergency department. Ann Emerg Med 2009;54(4):543-52.

8. American Academy of Pediatrics Committee on Pediatric Emergency Medicine, Frush K. Preparation for emergencies in the offices of pediatricians and pediatric primary care providers. Pediatrics 2007;120(1):200-12.

9. Santillanes G, Gausche-Hill M, Sosa B. Preparedness of selected pediatric offices to respond to critical emergencies in children. Pediatr Emerg Care 2006;22(11):694-8.

10. Jaimovich DG; Committee on Hospital Care and Section on Critical Care. Admission and discharge guidelines for the pediatric patient requiring intermediate care. Crit Care Med 2004;32(5):1215-8.

11. Thiriez G, Lefebvre A. Pediatric intermediate care demand, results of a survey in 3 French regions. Arch Pediatr 2010;17(8):1147-52. 


\section{Anexo 1 \\ GUÍA PARA CATEGORIZAR UCIP 1/ UCIP 2/ UR/ UCIM DE ACUERDO CON LA PRESENTE NORMA}

E: exigible.

D: deseable.

Institución

UCIP 1

UCIP 2

UR UCIM

\section{Personal}

Médicos

Jefe de Servicio de Cuidados Críticos.

Jefe de unidad o sala o coordinador de Cuidados Intensivos designado por autoridad hospitalaria apropiada, certificado en terapia intensiva pediátrica; horario mínimo de $6 \mathrm{~h}$ /día, $30 \mathrm{~h}$ semanales.

Un médico de planta certificado en terapia intensiva pediátrica cada 4 camas o fracción; horario mínimo de $6 \mathrm{~h} /$ día, 30 h semanales.

Un médico de planta certificado en terapia intensiva pediátrica cada 5 camas o fracción. Horario mínimo de $6 \mathrm{~h} /$ día, $30 \mathrm{~h}$ semanales.

Un médico de planta certificado en terapia intensiva pediátrica cada 8 camas o fracción. Horario mínimo de 6 h/día, 30 h semanales.

Médico de guardia las $24 \mathrm{~h}$ certificado en terapia intensiva pediátrica. Uno cada 6 camas.

Médico de guardia las $24 \mathrm{~h}$ con, al menos, 2 años de experiencia en terapia intensiva pediátrica certificada. Uno cada 6 camas.

Tercer médico de guardia (si se cuenta con especialista de pasiva), residente avanzado de otra especialidad, capacitado en emergencias pediátricas.

Médico de guardia las 24 h, capacitado en emergencias pediátricas.

$\mathrm{D}$

E $\quad$ E

E E

$\begin{array}{llll}\text { E } & \text { E } & & \text { E } \\ \text { E } & \text { E } & & \text { E } \\ \text { E } & \text { E } & & \\ & & & \text { E }\end{array}$

\section{Enfermería}

Jefe/a de enfermería, con un mínimo de 2 años de experiencia en terapia intensiva pediátrica; $36 \mathrm{~h}$ semanales.

Referente de enfermería: 1 por turno - $36 \mathrm{~h}$ semanales.

Enfermero/a de planta: $1 \mathrm{c} / 2$ camas $\mathrm{x}$ turno.

Enfermero de planta: $1 \mathrm{c} / 3-4$ camas $\mathrm{x}$ turno.

Enfermero/a de guardia activa.

\section{Otros}

Kinesiólogo de planta, al menos, 6 h/día.

E D D

Kinesiólogo de guardia activa las $24 \mathrm{~h}$ en el hospital.

E E

$\mathrm{D}$

Farmacéutico

Salud mental: psicólogo o psiquiatra disponible.

Personal de limpieza: $1 \mathrm{x}$ turno.

Personal de apoyo para tareas auxiliares: $1 \mathrm{x}$ turno.

Secretaría del servicio.

E $\quad$ E

E E

E D

E E

Especialidades y servicios de apoyo e interconsulta

Anestesiología con experiencia pediátrica con guardia activa de $24 \mathrm{~h}$.

Anestesiología con experiencia pediátrica con guardia pasiva de $24 \mathrm{~h}$.

Cirugía pediátrica: guardia activa o disponible dentro de $1 \mathrm{~h}$ si se cuenta con un cirujano general de guardia activa.

Neurocirugía: guardia activa de 24 h o disponible dentro de 1 hora.

Traumatólogo con guardia activa las 24 h; disponibilidad de consulta con traumatología pediátrica. 
Posibilidad de consulta con especialistas pediátricos no considerados en los ítems anteriores

Hemoterapia: guardia activa las $24 \mathrm{~h} \mathrm{c} /$ acceso a banco de sangre.

Radiología central con técnico de guardia activa las $24 \mathrm{~h}$.

Equipo de Rx portátil disponible para la unidad.

TAC dentro de la institución disponible las $24 \mathrm{~h}$.

TAC disponible las $24 \mathrm{~h}$.

Laboratorio activo las $24 \mathrm{~h}$.

RMN disponible en la institución o la posibilidad de realizarla.

Ecografía disponible dentro de los 60 minutos.

Radiología intervencionista de guardia pasiva las $24 \mathrm{~h}$.

$\begin{array}{lll}\text { E } & \text { E } & \text { E } \\ \text { E } & \text { E } & \text { E } \\ \text { E } & \text { E } & \text { E } \\ \text { E } & \text { E } & \text { E } \\ \text { E } & \text { E } & \text { D } \\ & & \text { E } \\ \text { E } & \text { E } & \text { E } \\ \text { E } & \text { E } & \text { E } \\ \text { E } & \text { E } & \text { E } \\ \text { E } & & \end{array}$

\section{Planta física}

No menor de 4-6 camas o 1000 días / pacientes por año.

No menor de 4 camas.

\section{Área de internación}

Stock de medicamentos.

E

Pileta o lavabo: mínimo 1 cada 4 camas; y gel-alcohol: uno por cama.

Ventanas.

Paredes (tratamiento especial).

Zócalo sanitario.

Salidas de oxígeno: 2 x cama.

Aspiración central: 1 x cama (preferentemente 2).

Aire comprimido: mínimo 1 x cama.

Iluminación general en el techo de aproximadamente 100 W; luz nocturna.

Luz intensa para procedimientos.

Puertas con abertura que permita el paso de camillas y equipos.

Visualización directa.

Sala de aislamiento.

Sistemas de alarma (alarma de paro).

Sistema eléctrico. 10 enchufes por cama. Enchufe para equipo rayos portátil.

Sistema eléctrico. 6 enchufes por cama. Enchufe para equipo rayos portátil.

Sistema de aire convenientemente filtrado y climatizado.

\section{Equipamiento}

Monitor multiparamétrico: 1 x cama (ECG, TA invasiva/no invasiva, temp., oximetría de pulso).

Monitor multiparamétrico: 1 x cama (ECG, TA no invasiva, oximetría de pulso).

Capnógrafo cada 4 pacientes en respirador.

Capnógrafo: 1 por unidad.

Respirador para la unidad (al menos, uno).

Respirador de alta frecuencia: al menos 1.

Carro de paro: según norma, 1 cada sector.

Bombas de infusión de precisión: 4 por cama (de 0,1 a $999 \mathrm{ml} /$ hora).

Bombas de infusión de precisión: 3 por cama (de 0,1 a $999 \mathrm{ml} /$ hora).

Bombas de infusión de precisión: 2 por cama (de 0,1 a $999 \mathrm{ml} /$ hora).

Bombas de alimentación: 1 cada dos camas.

$\begin{array}{llll}\text { E } & \text { E } & \text { E } & \text { E } \\ \text { E } & \text { E } & \text { E } & \text { E } \\ \text { D } & \text { D } & & \text { D } \\ \text { E } & \text { E } & \text { E } & \text { E } \\ \text { E } & \text { E } & \text { E } & \text { E } \\ \text { E } & \text { E } & \text { E } & \text { E } \\ \text { E } & \text { E } & \text { E } & \text { E } \\ \text { E } & \text { E } & \text { E } & \text { E } \\ & & & \\ \text { E } & \text { E } & \text { E } & \text { E } \\ \text { E } & \text { E } & \text { E } & \text { E } \\ \text { E } & \text { E } & & \text { E } \\ \text { E } & \text { E } & & \text { D } \\ \text { E } & \text { E } & \text { E } & \text { E } \\ \text { E } & \text { E } & & \text { E } \\ & & \text { E } & \\ \text { E } & \text { E } & \text { E } & \text { E }\end{array}$

E

E

E

\section{E}

E

E $\quad$ E

E $\quad$ E

E E

E

E

E

E $\quad$ E 
Electrocardiógrafo portátil: 1 por unidad.

E

Electrocardiógrafo portátil disponible.

Marcapasos externo transitorio: 1 cada 3 camas en CCV.

Marcapasos externo transitorio: 1 en la UCIP.

Bolsas de resucitación con reservorio y máscara: 1 por cama.

Equipo para cateterización venosa percutánea desde 4 Fr. hasta 7 Fr.: ( 2 x tamaño).

Equipo p/acceso intraóseo: 1 por unidad.

Equipo para medir VM.

Equipo p/disección venosa: 1 por unidad.

Equipo p/drenaje pleural.

Equipo para punciones (lumbar, abdominal).

Equipo p/ diálisis peritoneal.

Hemodiafiltración continua o equivalente.

Ecografía portátil disponible.

Ecocardiógrafo Doppler color disponible.

Ecografía Doppler transcraneana.

Equipo para regulación térmica del paciente.

Rx portátil disponible para la unidad.

Equipo para medir la presión intracraneana.

Cama/camilla transporte con monitor portátil multiparamétrico; tubo o $2 \mathrm{p} /$ transporte.

Sistema para prevención de escaras.

E

E

E

E

E

$\mathrm{D}$

E

E

E

E

E

E

E

$\mathrm{D}$

E

E

E

E

E
E

E

E

\section{Áreas de apoyo}

Estación de enfermería.

Sala de estar para enfermería y personal de limpieza.

Depósito de equipos.

Depósito de ropa y material de uso diario limpio.

Stock de medicamentos y material descartable.

Depósito de ropa y material sucio y de desecho.

Refrigerador para medicación.

Compartimento bajo llave para narcóticos y electrolitos de alta concentración.

Refrigerador independiente para fórmulas de alimentación.

Depósito de ropa y material sucio y de desecho.

Área de lavado y preparación para material quirúrgico.

Área administrativa y/o de secretaría.

Área de recepción de familiares.

Área de reunión de médicos.

Oficina del jefe médico y de enfermería.

Habitación para médicos de guardia.

Baños para personal.

Chatero.

Medidas de protección contra incendios, plan de evacuación.

Acceso a internet, biblioteca para consultas médicas.

\section{Registros}

Diagnóstico, fecha de ingreso y egreso, datos demográficos.

Tasa de mortalidad estandarizada.

Condiciones de egreso.

Infecciones adquiridas en la unidad.

Eventos adversos, extubación no programada, caídas de la cama, úlceras por decúbito.

Al menos, 1 indicador de procesos.

E

$$
\mathrm{D}
$$$$
\text { E E E }
$$$$
\text { E E }
$$$$
\text { E E E }
$$$$
\mathrm{D}
$$$$
\text { E }
$$$$
\text { E }
$$$$
\text { E }
$$$$
\text { E }
$$$$
\text { D }
$$$$
\text { E }
$$$$
\text { E }
$$$$
\text { E }
$$$$
\text { E E }
$$$$
\text { E E }
$$$$
\text { E }
$$

E D

\begin{tabular}{|c|c|c|}
\hline $\mathrm{E}$ & & $\mathrm{E}$ \\
\hline $\mathrm{E}$ & & E \\
\hline $\mathrm{E}$ & & $\mathrm{E}$ \\
\hline $\mathrm{E}$ & & $\mathrm{E}$ \\
\hline $\mathrm{E}$ & $\mathrm{E}$ & $\mathrm{E}$ \\
\hline $\mathrm{E}$ & & $\mathrm{E}$ \\
\hline $\mathrm{E}$ & $\mathrm{E}$ & $\mathrm{E}$ \\
\hline $\mathrm{E}$ & $\mathrm{E}$ & E \\
\hline $\mathrm{E}$ & & $\mathrm{E}$ \\
\hline $\mathrm{E}$ & & $\mathrm{E}$ \\
\hline $\mathrm{E}$ & & $\mathrm{E}$ \\
\hline $\mathrm{E}$ & & $\mathrm{E}$ \\
\hline $\mathrm{D}$ & & $\mathrm{E}$ \\
\hline $\mathrm{E}$ & & E \\
\hline $\mathrm{E}$ & & E \\
\hline $\mathrm{E}$ & & $\mathrm{E}$ \\
\hline $\mathrm{E}$ & & E \\
\hline $\mathrm{E}$ & & $\mathrm{E}$ \\
\hline $\mathrm{E}$ & & $\mathrm{E}$ \\
\hline $\mathrm{E}$ & & $\mathrm{E}$ \\
\hline $\mathrm{E}$ & $\mathrm{E}$ & $\mathrm{E}$ \\
\hline $\mathrm{E}$ & & \\
\hline $\mathrm{E}$ & & $\mathrm{E}$ \\
\hline $\mathrm{E}$ & & \\
\hline $\mathrm{E}$ & & E \\
\hline E & & \\
\hline
\end{tabular}


IV / Arch Argent Pediatr 2413;112(3):287-293 / Subcomisiones, Comités y Grupos de Trabajo

\section{Normas de procedimientos y tratamiento}

Guías de diagnóstico y tratamiento (patología habitual).

Protocolos de atención de enfermería.

Programa de mejora de calidad y atención segura de pacientes.

Política de consentimiento informado.

Programa de control de infecciones.

Protocolo para procedimientos invasivos.

Normas de atención ante paro cardiorrespiratorio.

\begin{tabular}{llll} 
E & E & & E \\
E & E & & E \\
E & E & & D \\
E & E & E & E \\
E & E & & E \\
E & E & E & E \\
E & E & E & E \\
\hline
\end{tabular}

